\title{
MONITORING SLEEP DEPTH: ANALYSIS OF BISPECTRAL INDEX (BIS) BASED ON POLYSOMNOGRAPHIC RECORDINGS AND SLEEP DEPRIVATION
}

Sandra Giménez $z^{1,2,3,7^{*}}$, Sergio Romero ${ }^{4,5 *}$, Joan Francesc Alonso ${ }^{4,5,6}$, Miguel Ángel Mañanas ${ }^{4,5,6}$, Anna Pujol ${ }^{8}$, Pilar Baxarias ${ }^{8}$, Rosa Maria Antonijoan ${ }^{1,2,7}$

1. Drug Research Center, Hospital de la Santa Creu i Sant Pau, Barcelona, Spain.

2. Department of Pharmacology and Therapeutics, Universitat Autònoma de Barcelona (UAB), Barcelona, Spain.

3. Sleep Unit. Respiratory Department. Hospital de la Santa Creu i Sant Pau. Barcelona, Spain.

4. Biomedical Engineering Research Centre, Department of Automatic Control, Universitat Politècnica de Catalunya. BarcelonaTech (UPC), Barcelona, Spain.

5. CIBER de Bioingeniería, Biomateriales y Nanomedicina (CIBER-BBN), Spain.

6. Barcelona College of Industrial Engineering (EUETIB), UPC, Barcelona, Spain.

7. CIBER de Salud Mental (CIBERSAM), Spain.

8. Anesthesiology Service. Fundació Puigvert, Barcelona, Spain.

* S. Giménez and S. Romero contributed equally to this work.

\begin{abstract}
The assessment and management of sleep are increasingly recommended in the clinical practice. Polysomnography (PSG) is considered the gold standard test to monitor sleep objectively, but some practical and technical constraints exist due to environmental and patient considerations. Bispectral index (BIS) monitoring is commonly used in clinical practice for guiding anesthetic administration and provides an index based on relationships between EEG components. Due to similarities in EEG synchronization between anesthesia and sleep, several studies have assessed BIS as a sleep monitor with contradictory results. The aim of this study was to evaluate objectively both the feasibility and reliability of BIS for sleep monitoring through a robust methodology, which included full PSG recordings at a baseline situation and after $40 \mathrm{~h}$ of sleep deprivation. Results confirmed that the BIS index was highly correlated with the hypnogram $(0.89 \pm 0.02)$, showing a progressive decrease as sleep deepened, and an increase during REM sleep (awake: $91.77 \pm 8.42$; stage N1: 83.95 \pm 11.05 ; stage N2: 71.71 \pm 11.99 ; stage N3: 42.41 \pm 9.14 ; REM: $80.11 \pm 8.73$ ). Mean and median BIS values were lower in the postdeprivation night than in the baseline night, showing statistical differences for the slow wave sleep (baseline: $42.41 \pm 9.14$ vs post-deprivation: $39.49 \pm 10.27$; $\mathrm{p}=0.02$ ). BIS scores were able to discriminate properly between deep (N3) and light (N1, N2) sleep. BIS values during REM overlapped those of other sleep stages, although EMG activity provided by the BIS monitor could help to identify REM sleep if needed. In conclusion, BIS monitors could provide a useful measure of sleep depth in especially particular situations such as intensive care units, and they could be used as an alternative for sleep monitoring in order to reduce PSG-derived costs and to increase capacity in ambulatory care.
\end{abstract}

Keywords: Polysomnogram, sleep depth, BIS monitor, slow wave activity, approximate entropy. 


\section{INTRODUCTION}

Polysomnography (PSG) constitutes the gold standard technique for assessing sleep and diagnosing its related disorders, and provides extensive information about sleep behavior and physiology. However, due to its requirements in terms of time, equipment, economic costs, and personnel, PSG may be too complex and invasive to be used in clinical studies focused simply on the quantification of sleep time, wake time or both.

Intensive Care Units (ICU) constitute one of these cases where environmental requirements and patient condition make PSG technically unfeasible. In fact, it is known that the particular situation of ICU patients, who are often sleep deprived and present disrupted sleep architecture and poor sleep quality, affects negatively on their recovery [1,2]. Since the Society of Critical Care Medicine recommended that sleep assessment should be performed mainly to prevent consequences on cognitive function, alternative measures of sleep to PSG have been investigated, but some options such as actigraphy or subjective assessment have resulted to be inaccurate and misleading [3].

On the other hand, certain clinical situations demand real-time sleep stage identification. For example, REM sleep deprivation is used as a treatment in some andrological post-surgeries to avoid REM-related erections, and several works have also shown the usefulness of REM sleep restriction in the treatment of depression [4,5].

In the last decades, EEG-based devices have been developed for monitoring sedation under anesthesia or in critical care environments. Taking into account similarities between sleep and anesthesia, a reasonable question is to investigate if these depth-of-anesthesia monitors could also be considered as depth-of-natural-sleep monitoring devices. Among the available alternatives, the Bispectral Index (BIS) monitor has been examined for usefulness as a sleep stager. The BIS monitor was developed to assess changes in brain activity throughout the course of anesthetic or sedative administration, providing a measure of hypnotic effects. The monitor processes the EEG data acquired by sensors placed in the forehead and calculates an index by combining selected features in time and frequency domains [6]. The BIS index indicates the level of sedation that correlates to the clinical effects of anesthesia, quantifying the degree of coherence among different frequencies in the EEG signal on a scale of 0 to 100 . Values around 100 represent wakefulness (good cortical integration), while an increase in the depth of anesthesia results in a decreased cortical integration and thus lower BIS scores. Values in the range of 40 to 60 indicate adequate hypnotic effects during general anesthesia.

Different works have assessed the effectiveness of BIS in monitoring natural sleep but this relationship is still unclear. Most studies have demonstrated a decrease in BIS scores with increasing sleep depth during slow-wave sleep, but with discrepancies about the correlation between BIS data and sleep stages [7,8]. Besides, all of the previous BIS-studies share a substantial limitation because none of them performed a full PSG study, the gold-standard reference for sleep assessment using electrooculographic (EOG), electroencephalographic (EEG) and electromyographic (EMG) channels, against which BIS data could have been compared and validated.

Before BIS monitoring can be used regularly in clinical studies or applied to special populations, it would be desirable to confirm its reliability as a monitor of sleep depth and as a sleep stager in physiological healthy subjects. This would provide the tools for the definition and comparison of sleep research protocols. Hence, the main aim of this study was to evaluate the performance of BIS as a sleep monitor, analyzing its benefits and limitations. Using a robust methodology based on full PSG recordings from 40-hour sleep-deprived volunteers, the information provided by BIS was assessed to evaluate its ability to identify sleep stages. 


\section{METHODS}

\section{Study design}

Twelve healthy male young adult volunteers (mean age 27.75 years, range 21-34) were recruited for the study from the pool of volunteers at the Pharmacology Research Unit (CIM) of the Hospital de la Santa Creu i Sant Pau in Barcelona, Spain. Medical interviews and examinations, clinical chemistry, hematology, and urinalysis tests were performed within 21 days before the start of the study. Exclusion criteria included any history of medical or psychiatric illnesses and screening for subjective sleep disturbances (Pittsburgh Sleep Quality index $<5$ ). The first PSG night, in addition to familiarization purposes, allowed to exclude any undiagnosed sleep disorder objectively $(\mathrm{AHI}<5 / \mathrm{h} ; \mathrm{PLMI}<5 / \mathrm{h})$.

Participants were not allowed to take any medication during the study without the prior knowledge of the research team and were asked to keep regular sleep-wake habits in the month before the study initiation and during the study itself. The latter was verified by checking individual sleep diaries.

The trial was approved by the local Ethics Committee and was conducted following the principles stated in the Declaration of Helsinki and the guidelines of Good Clinical Practice. The study was registered by the Biomedical Research Institute of the Hospital of Sant Pau with the number CIM/04/213/01: "Evaluation of the bispectral index (BIS) in the different sleep stages in baseline and after sleep deprivation". All volunteers gave written informed consent before the start of the study and were paid for their participation. The study was conducted according to an intervention-controlled, crossover design. All volunteers completed the study which included 3 PSG nights: one adaptation night (PSG1), one baseline night (PSG2) and one recovery night after 40 hours of sleep deprivation (PSG3). For the sleep deprivation experimental session, subjects arrived at 07:00 am and stayed awake for 40 hours in the laboratory. Volunteers were under continuous direct supervision of the staff and were not allowed to nap. Figure 1 shows the schematic diagram of the experimental trial.

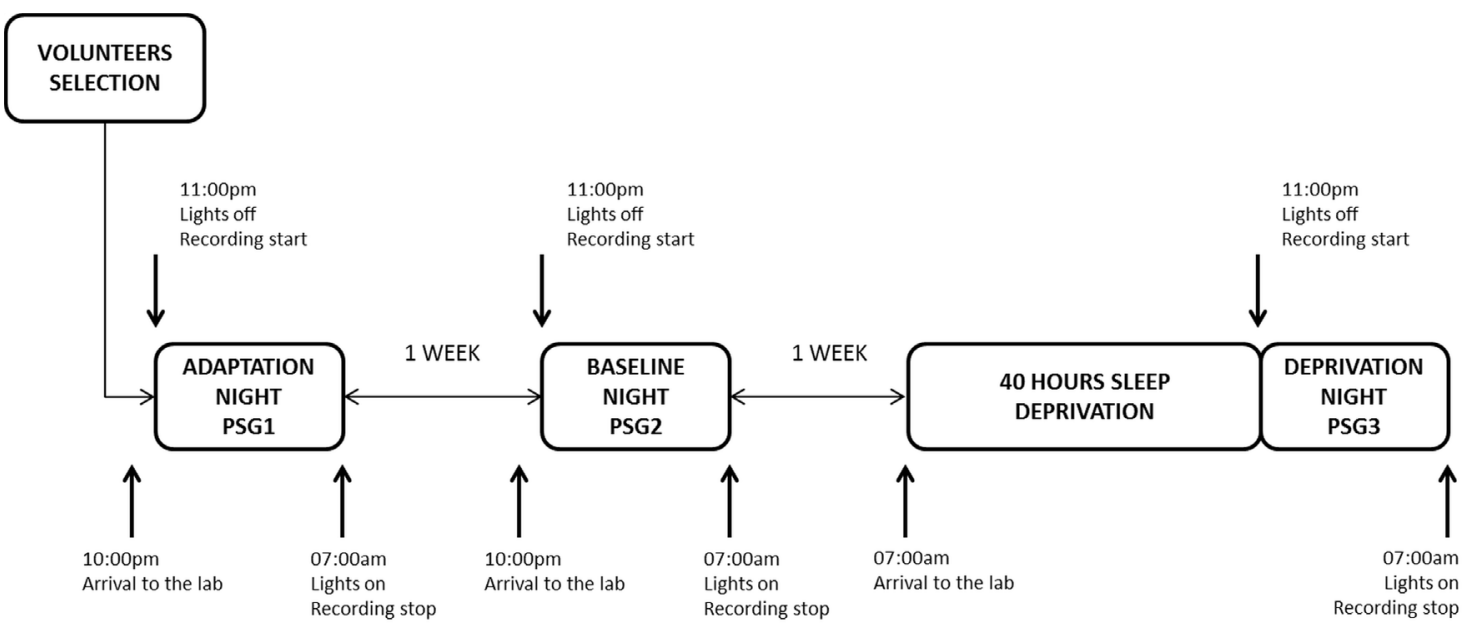

Fig. 1: Schematic diagram of the planning of the deprivation study indicating the nights with polysomnography recording and the washout time between them.

\section{PSG recordings and sleep stage classification}

Sleep recordings were performed in individual, sound-attenuated, temperature-regulated rooms and volunteers were supervised by qualified technical staff. Sleep EEG data was acquired by means of the Compumedics system. The recordings consisted of 6 EEG channels (Fp1, Fp2, 
$\mathrm{C} 3, \mathrm{C} 4, \mathrm{O} 1, \mathrm{O} 2$ referenced to averaged mastoid (A1-A2) according to the international 10-20 system), 2 electrooculographic (EOG) channels and 1 chin electromyographic channel (EMG). Four channels were included to monitor respiratory function, 1 for the airflow signal, 2 channels to record rib cage and abdominal motion, and the last for oxygen saturation. Heart rate variability was controlled by means of an ECG channel. Finally, limb movements were monitored using 2 channels with linked electrodes on both right and left anterior tibialis. EEG, EOG, ECG and EMG signals were acquired with a sampling frequency of $256 \mathrm{~Hz}$ and channels for respiratory function were recorded using a sample rate of $25.6 \mathrm{~Hz}$. EOG, EEG and ECG channels were filtered to a bandwidth of 0.1 to $75 \mathrm{~Hz}$. For EMG channels the bandwidth 10 to $75 \mathrm{~Hz}$ was used. Channels were calibrated before each recording and the electrode impedance was kept below $10 \mathrm{k} \Omega$. A notch filter was applied to reject $50 \mathrm{~Hz}$ electrical noise.

The sleep stages were visually scored for 30-s epochs according to the new guidelines of the American Academy of Sleep Medicine (AASM) [9] using the Profusion Sleep Software (Compumedics ${ }^{\circledR}$ PSG3 version 3.4). Scoring was performed by two independent sleep experts. Discrepancies were solved by a third expert from the same laboratory. Derived sleep variables were classified into two groups: 1) sleep initiation and maintenance (total sleep time, sleep efficiency, different stage latencies and wake time); and (2) sleep architecture (time and percentages of the different stages: stage N1, stage N2, stage N3 or SWS, and stage R or REM sleep). Sleep onset latency was considered as the time taken to reach sleep stage N2.

\section{EEG analysis}

EEG signals were segmented into epochs of 5 seconds. Artifacted epochs containing either saturation or muscular activity were automatically identified and removed from the analysis. Power spectra of artifact-free epochs were performed by means of the Welch's periodogram. Dynamics of slow wave activity (SWA), calculated as the absolute power in the band 0.5 to 4.0 $\mathrm{Hz}$, was computed throughout the whole night. Approximate entropy (ApEn), which is a measure of signal irregularity, was also calculated for the artifact-free epochs. Entropy measurement has shown its usefulness for the estimation of sleep stages and the representation of sleep as a continuum process $[10,11]$.

\section{BIS recordings}

BIS data were collected by using BIS Quatro Sensors placed on the forehead and measured by an A-2000 XP Monitor. BIS data was extracted every 5 seconds simultaneously with PSG data using the RS232 output of the BIS monitor and recorded into an ASCII file in the computer. Recorded data comprised, among others, electrode impedances, signal quality index (SQI), BIS value, and muscle activity measured as electromyography (EMG) in dB. Fivesecond segments of BIS data with values of SQI less than $50 \%$ were not included in the analysis. Because BIS data was collected every 5 seconds and experts classified sleep stages every 30 seconds, the sleep stage scores were replicated in 6 windows of 5 seconds in order to compare BIS values with sleep stage classification. For example, if a 30 s epoch was classified as stage N2, all the $5 \mathrm{~s}$ windows of this 30 s epoch were labelled as stage N2.

\section{Statistical analysis}

Mean values of PSG and BIS derived parameters were calculated for each sleep stage: W, N1, N2, N3 (SWS) and R separately for each night, baseline and post-deprivation. Sleep deprivation effects were assessed by paired 2-tailed t-tests to compare sleep variables obtained in the post-deprivation night (PSG3) with respect to the baseline night (PSG2). Statistical significance was set to 0.05 . Similarity between the waveforms was calculated by normalized cross-correlation between the hypnogram and the time courses of different variables (BIS, SWA, ApEn) taking into account both nights. 


\section{RESULTS}

Table 1 shows the PSG variables related to sleep initiation and maintenance, and sleep architecture calculated in the baseline and post-deprivation conditions. As expected, sleep onset calculated as the latency to stage N2 was statistically shorter during the post-deprivation night compared with the baseline night $(\mathrm{p}=0.002)$. Statistical significant increases in total sleep time $(p=0.003)$, sleep efficiency $(p=0.002)$ and SWS $(p=0.001)$ were also observed after sleep deprivation.

Table 1. Classical PSG variables related to sleep initiation and maintenance and sleep architecture in both conditions: baseline and post-deprivation nights.

\begin{tabular}{|c|c|c|c|c|c|}
\hline \multicolumn{3}{|c|}{ Sleep initiation and maintenance } & \multicolumn{3}{|c|}{ Sleep architecture } \\
\hline & Baseline & Post-deprivation & & Baseline & Post-deprivation \\
\hline Latency to stage N1 (min) & $21.2 \pm 32.1$ & $14.1 \pm 30.7$ & Stage N1 (min) & $17.8 \pm 7.4$ & $7.5 \pm 6.2^{* *}$ \\
\hline Latency to stage N2 (min) & $28.4 \pm 31.7$ & $6.8 \pm 11.2^{* *}$ & Stage N1 (\%) & $4.6 \pm 2.3$ & $1.7 \pm 1.4^{* *}$ \\
\hline Latency to stage N3 (min) & $23.0 \pm 28.0$ & $8.1 \pm 2.8^{*}$ & Stage N2 (min) & $236.7 \pm 57.1$ & $241.0 \pm 30.1$ \\
\hline REM latency (min) & $113.6 \pm 42.9$ & $110.7 \pm 39.5$ & Stage N2 (\%) & $58.5 \pm 6.6$ & $52.1 \pm 6.4^{* *}$ \\
\hline Total Sleep Time (min) & $401.8 \pm 71.3$ & $462.8 \pm 21.4^{* *}$ & Stage N3 (min) & $77.7 \pm 28.4$ & $127.1 \pm 33.1^{* *}$ \\
\hline Sleep efficiency (\%) & $83.6 \pm 14.7$ & $96.4 \pm 4.4^{* *}$ & Stage N3 (\%) & $20.0 \pm 8.4$ & $27.4 \pm 6.9^{* *}$ \\
\hline Wake time (min) & $73.9 \pm 70.8$ & $23.9 \pm 32.3^{*}$ & REM (min) & $67.8 \pm 27.8$ & $84.2 \pm 24.3^{*}$ \\
\hline Wake/TST (\%) & $15.4 \pm 14.8$ & $5.0 \pm 6.7^{*}$ & REM (\%) & $16.4 \pm 5.0$ & $18.2 \pm 5.1$ \\
\hline
\end{tabular}

Figure 2 shows, as an example, a sleep hypnogram compared with the time course of the BIS index for one volunteer in both conditions: baseline and post-deprivation. The information of the time course of SWA and ApEn computed from the channel C4 were included in the figure. BIS values, as well as SWA and entropy, appeared to follow the morphology of the hypnogram. As expected, SWA increased and entropy decreased in the periods of deep sleep.

In non-REM sleep (NREM), EEG becomes slower and more synchronized in the transient from stage N1 to stage N3 (SWS). This effect could be observed in the time course of entropy, which reached the lowest values (EEG signal more regular) when the maximum level of synchronization was achieved in SWS. High values of correlation were obtained between hypnogram and the time courses of all variables: hypnogram vs BIS: $0.891 \pm 0.019$; hypnogram vs SWA: $0.669 \pm 0.084$; hypnogram $v s$ ApEn: $0.873 \pm 0.027$. The highest correlation values were obtained for BIS index and approximate entropy.

On the other hand, an increase in the variability could be clearly seen in certain intervals of the time course of the EMG activity acquired by the BIS monitor. These intervals corresponded to REM sleep and were visually detected and marked as black lines in Figure 2.

BIS values corresponding to sleep onset were calculated for all volunteers, and no significant differences were obtained between the baseline and the post-deprivation nights: 83.5 \pm 6.5 and $82.6 \pm 5.4$, respectively.

Figure 3 shows the distribution of BIS values according to the sleep stage for both conditions: baseline and post-deprivation night (black and grey traces, respectively). BIS values showed a progressive decrease with the sleep depth. High inter-subject correlations of average BIS index were observed with wakefulness and NREM stages for both conditions $\left(\mathrm{R}^{2}=0.912\right.$ for baseline and $\mathrm{R}^{2}=0.945$ for post-deprivation night). The overlap among boxplots indicated that BIS could not differentiate accurately between some sleep stages, especially if they were consecutive. However, clear differences in BIS were recognized between light sleep (stages N1 and N2) and slow wave sleep (stage N3), but BIS values during REM could be easily confused with sleep stages $\mathrm{N} 1$ and $\mathrm{N} 2$. 

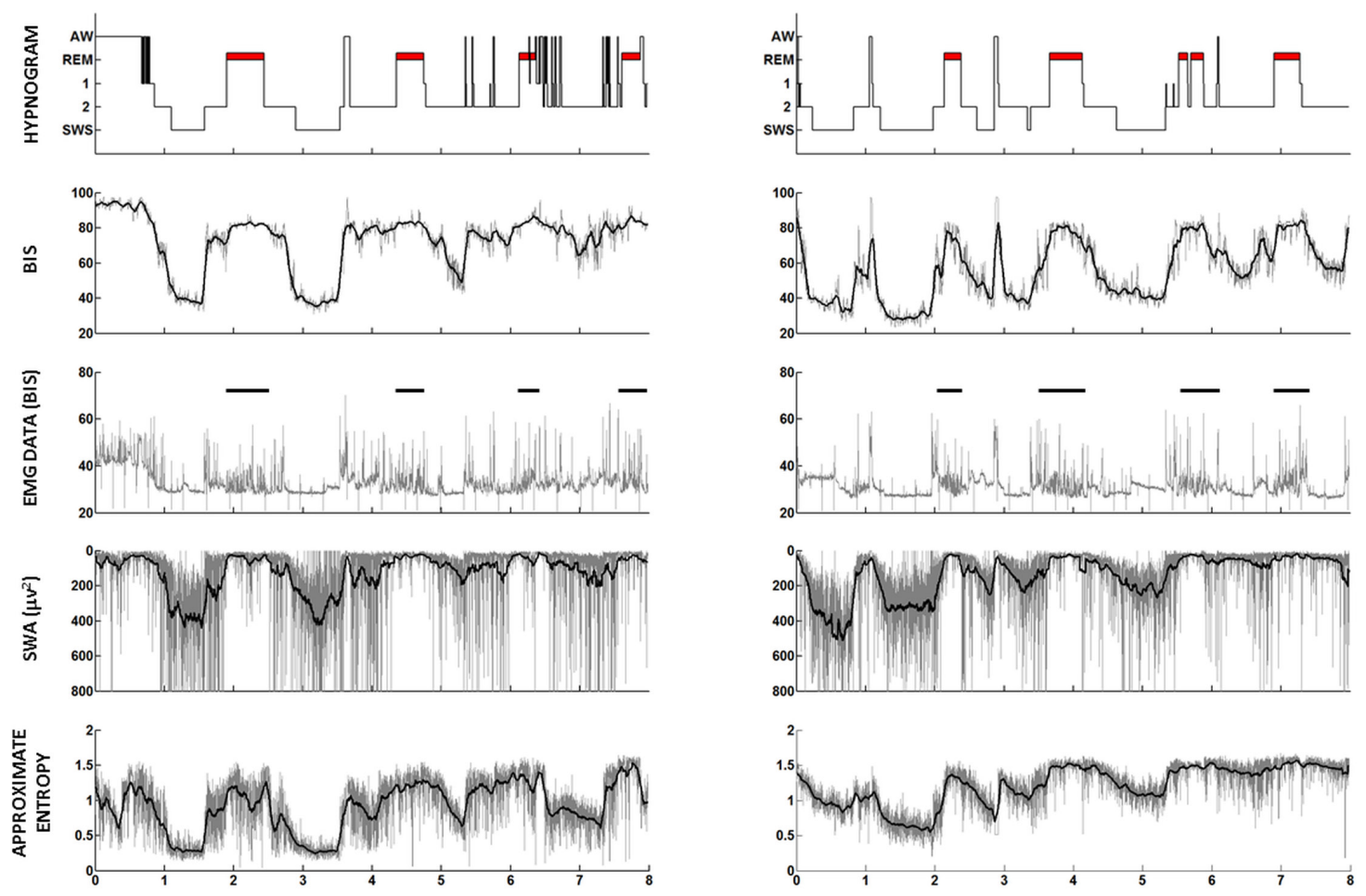

Fig. 2: Time course of the hypnogram, BIS and EMG data extracted from the BIS monitor, slow wave activity (SWA; 0.5 to $4.0 \mathrm{~Hz}$ ), and approximate entropy obtained for a subject as an example. For each boxplot, the central mark is the median, the edges of the boxes account for the 25th and 75th percentiles, and the whiskers extend to the most extreme BIS data points considering coverage of $99 \%$. Left column corresponds to the baseline night and right column to the night after 40 hours of sleep deprivation. In the hypnogram, AW refers to stage W, REM to stage R, 1 to N1, 2 to N2 and SWS to stage N3. Thick lines in the graphs corresponding to BIS, SWA and approximate entropy were calculated by means of a moving average filter using a window of 5 minutes.

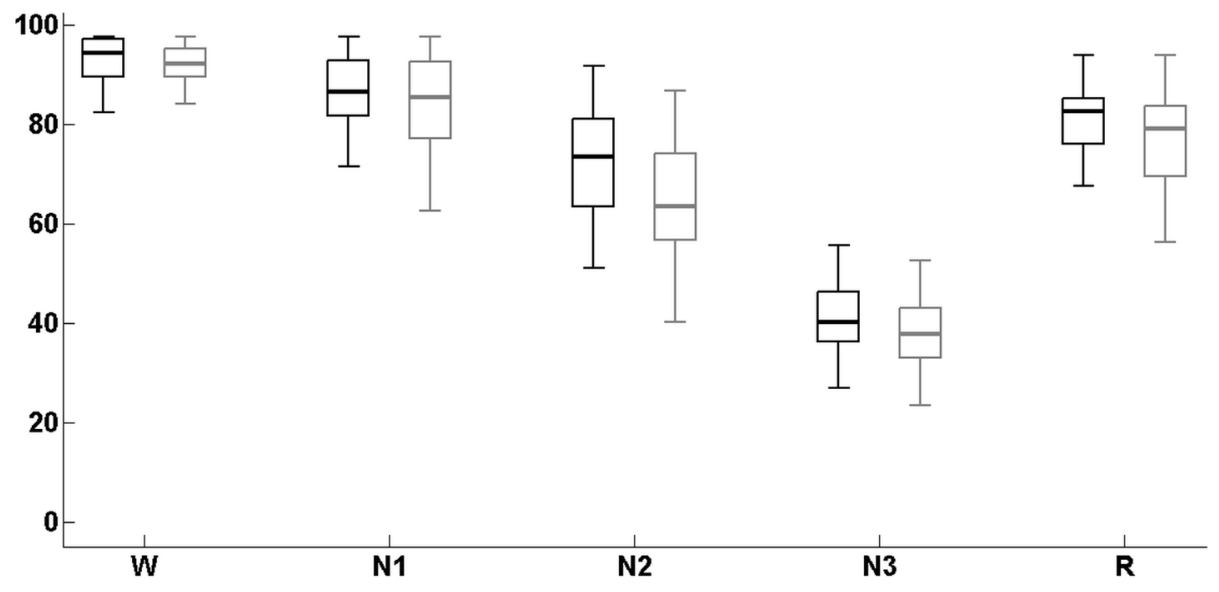

Fig. 3: Boxplots showing the distribution of BIS values depending on the sleep stage. The central mark of each box corresponds to the median, the edges of the box are the 25th and 75th percentiles and the whiskers extend to the extreme data points (98\%) not considering outliers. Black and grey boxplots indicate the baseline night and the postdeprivation night, respectively.

No significant differences in mean BIS values were observed between both conditions (baseline and post-deprivation) in any sleep stage, except for the stage N3 (SWS). Figure 4 
shows the mean BIS values during SWS as a function of NREM sleep cycles. Mean values during SWS calculated from all night were significantly lower after sleep deprivation than in the baseline night $(\mathrm{p}=0.021)$. Attending to the first three sleep cycles, differences were observed in the first NREM cycle $(\mathrm{p}<0.001)$ whereas the second and third cycles did not show significant changes ( $\mathrm{p}=0.631$ and $\mathrm{p}=0.532$, respectively).

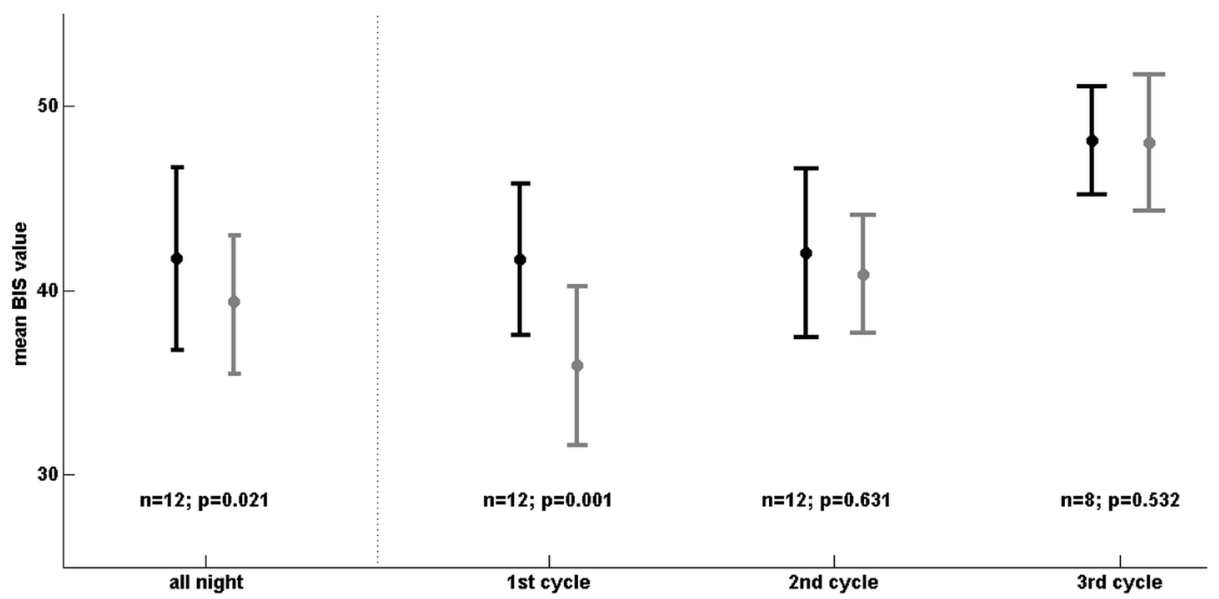

Fig. 4: Mean BIS values calculated for 12 subjects using all-night data, and data of the different NREM sleep cycles. Black and grey traces correspond to the baseline night and night after $40 \mathrm{~h}$ of sleep deprivation, respectively.

Figure 5 shows the probability of belonging to a given sleep stage by means of the BIS value. This figure indicates that very high BIS values (above 95) implicated wakefulness and low BIS values (below 40) involved SWS. Maximum probability of light sleep (stages N1 and N2) was for BIS indexes between 55 and 80, whereas REM sleep was most likely indicated by BIS values between 80 and 90 .
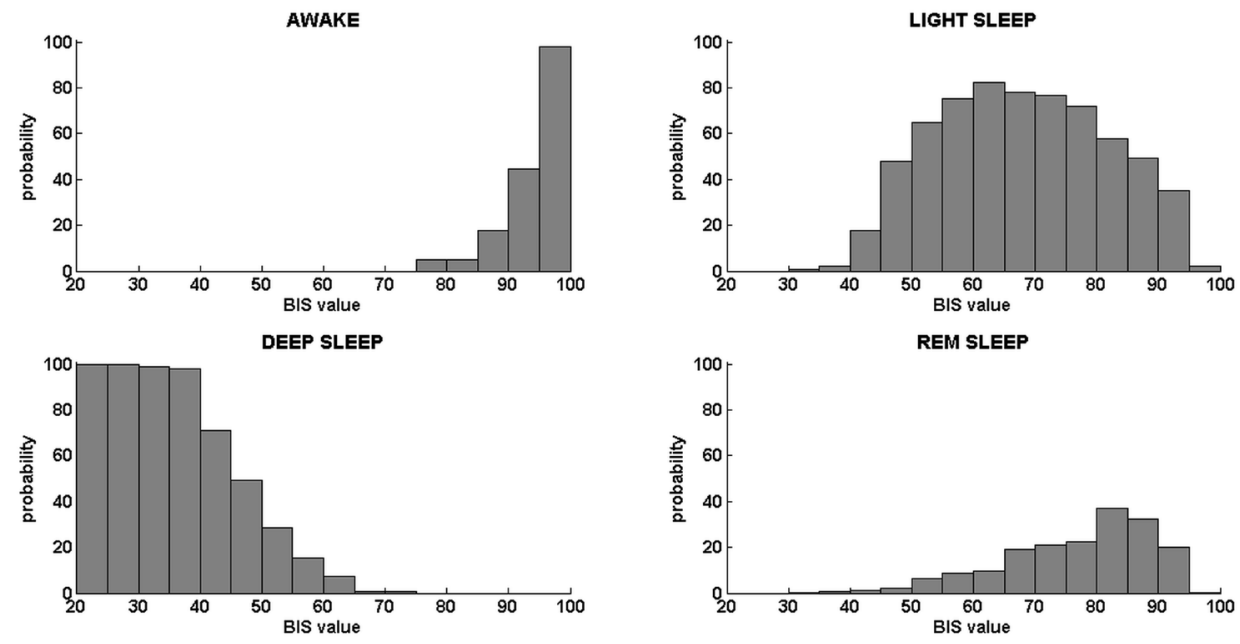

Fig. 5: Probability of being in a particular sleep state given a BIS value. These probabilities were calculated from all the data of the study: all volunteers and both nights: baseline and post-deprivation. Light sleep refers to stages N1 and N2, and deep sleep refers to stage N3 or SWS. 


\section{DISCUSSION}

This study evaluated the benefits and limitations of BIS scoring as a tool for monitoring sleep depth and sleep stages, comparing the BIS index with full PSG data in healthy volunteers under sleep deprivation. Results showed that BIS scores changed during physiological sleep: decreasing during NREM sleep with increasing depth of sleep (from stage N1 to stage N3), showing a highly correlated course with the hypnogram, SWA dynamics, and spectral entropy. BIS index increased during REM sleep, overlapping with the values obtained for sleep stages $\mathrm{N} 1$ and N2. Mean and median BIS values were lower in the post-deprivation night in comparison to the baseline night, showing statistical differences during the slow wave sleep (stage N3).

Anesthesia and natural sleep show similar features but act via different physiological mechanisms. Anesthesia and other mechanisms that reduce the level of consciousness typically produce a progressive increase of low-frequency EEG, high-amplitude activity as anesthesia deepens, rather resembling the spontaneous activity occurring during sleep $[12,13]$. Therefore, it is not strange that several studies have assessed BIS as a sleep monitor, but contradictory and non-conclusive results have been obtained [7,8].

Deep sleep is characterized by the hyperpolarization of the thalamocortical membrane potentials, a phenomenon that is translated into EEG slow waves of fairly high amplitude, causing an increase in the slow activity and in the regularity of the EEG signals. It is important to stress that BIS index decreased with depth of sleep for all subjects in both nights, reproducing the sleep architecture obtained by concomitant PSG recordings. All BIS scores were lower during sleep than during wakefulness, with minimal scores corresponding to SWS. The night after $40 \mathrm{~h}$ of sleep deprivation BIS scores were reduced compared to the baseline night, showing especially significant decreases during the stage $\mathrm{N} 3(\mathrm{p}=0.021)$. These results showed that BIS was able to follow EEG changes occurring after sleep deprivation. According to the two-process model of sleep regulation, sleep pressure accumulates during wakefulness and dissipates in the course of the following sleep episode [14], and slow wave activity (SWA) during NREM sleep is the key electrophysiological marker of this homeostatic process. It has been widely demonstrated that sleep deprivation increases the homeostatic sleep pressure, causing an increase in SWS and SWA in the following recovery sleep night that is more pronounced in the first sleep cycle [15]. In our study, we found BIS indexes correlated with SWA and followed closely the dynamics of this frequency range along the night, with minimum scores obtained for the first NREM sleep cycle $(\mathrm{p}=0.001)$.

The distribution and percentages of the sleep stages across the night is diverse: wakefulness constitutes approximately $5 \%$ of the night, stage N1 accounts for less than $5 \%$, stage N2 covers $45 \%$ to $55 \%$ of sleep, stage N3 comprises about $15 \%$ to $25 \%$, and REM sleep is usually $20 \%$ to $25 \%$ of sleep in typical healthy adults [16]. Similar values were obtained in our study for the baseline and post-deprivation nights (see Table 1). These different percentages affect the probability of given a BIS value to belong a particular sleep stage. For example, BIS values comprised between 70 and 80 are more probable to be linked to stage N2 than REM, because stage N2 is more predominant than REM in the night (see Figure 5).

Surprisingly, only one study assessed the temporal reduction in BIS values after sleep deprivation, which guaranteed a reliable marker of sleep depth [17]. In this study, Dahaba et al. nocturnal sleep was completely not assessed since volunteers went to sleep at 8 am which could interfere with circadian sleep regulation. Moreover, preprocessed EEG data were extracted from BIS sensors including information just from frontal locations in contrast to our full 10-20 montage. Nevertheless, mean BIS values for the different sleep stages after sleep deprivation obtained in Dahaba et al. were similar to ours (reaching minimal BIS scores close to 40 in both studies). Similarities between both studies enhanced the strength of BIS monitor to measure sleep depth. 
Several studies have criticized AASM rules because of the low temporal resolution achieved using epochs of 30 seconds [18,19]. The biological sleep process follows a continuous course in which soft transitions appear between different sleep stages. Overall, BIS scores can be considered a pseudo-continuous measure of the sleep process because BIS A2000-XP monitor uses 5-second windows and a scale ranging from 0 to 100 to describe sleep states.

On the other hand, the sensitivity of BIS monitoring to detect transitions from wakefulness to sleep in healthy volunteers has already been assessed, concluding that a threshold BIS value of 80 could be set to detect sleep onset although sleep onset latency was evaluated subjectively [20]. However, we calculated sleep onset objectively by means of PSG. Similar threshold BIS values for sleep onset were observed in our study (around 82) but there was variability among volunteers: while a volunteer could reach a first epoch of stage N2 with a BIS score around 75, another could reach stage N2 with a BIS value around 90.

Contrary to NREM sleep, wakefulness and REM stages are associated to a higher cortical function, exhibiting cortical desynchronization and indeed less coherence. The BIS values obtained in this study during REM sleep increased, showing an overlap with light sleep scores (stages N1 and N2). However, EMG activity provided by BIS monitors for detecting artifacts, clearly matched REM periods and helped to identify this stage. The increase of variability of EMG activity during REM sleep could be originated by the ocular activity or the characteristic phasic muscular twitches.

Literature also reports a direct relationship between changes in the BIS values during physiological sleep and the brain activity measured by Positron Emission Tomography (PET). Specifically, the activity of specific cortical (frontoparietal network) and subcortical (brainstem and thalamus) areas, involved in the content of consciousness and the modulation of arousal, respectively, has been shown to decrease progressively with sleep depth and to return to values close to awake in REM sleep, showing a positive correlation with the BIS index [21]. Moreover, other PET studies have shown that the decline of regional cerebral blood flow during SWA is most prominent in frontal cortical areas [22,23]. Consequently, BIS monitoring should be theoretically optimal with respect to the location of electrodes (frontal areas) and this could represent a simpler index of cerebral activity.

Some limitations of this research merit further consideration. BIS data was recorded by a first-generation BIS Monitor (A2000-XP) and there have been different software and hardware updates during the last decade that have given rise to the latest model of BIS monitor (BISVista). Although no statistical differences in mean BIS values were found between the two monitors, BIS-Vista had a lower variance than the A2000 thanks to improvements in the filtering of electromyographic artifacts [24]. Finally, future studies in clinical environments where PSG recordings are technically difficult (such as intensive care units) should complement the feasibility and reliability of BIS index as a marker of sleep depth.

In the current study, the performance of BIS index showed sleep architecture patterns compatible with the gold standard reference for sleep monitoring (PSG) that evidenced the potential use of BIS scoring as a marker of sleep depth and for differentiating between some sleep stages. The variability of EMG activity, supplied by BIS monitors, can be also useful to identify REM sleep.

\section{ACKNOWLEDGEMENTS}

The authors thank the staff at the Centre d'Investigació de Medicaments de l'Institut de Recerca de l'Hospital de la Santa Creu i Sant Pau for their technical assistance during data collection. The present research complies with the Spanish Law. 


\section{DISCLOSURE OF POTENTIAL CONFLICT OF INTEREST}

This study was supported in part by the Ministerio de Economía y Competividad, under contract DPI2011-22680 and DPI2014-59049R. CIBER-BBN and CIBERSAM are an initiative of the Instituto de Salud Carlos III, Spain. The authors declare that there is no conflict of interest arising from this study.

\section{REFERENCES}

1. Friese RS, Diaz-Arrastia R, McBride D, Frankel H, Gentilello LM. Quantity and quality of sleep in the surgical intensive care unit: are our patients sleeping? J. Trauma. 2007;63:1210-4.

2. Weinhouse GL, Schwab RJ. Sleep in the critically ill patient. Sleep. 2006;29:707-16.

3. Bourne RS, Minelli C, Mills GH, Kandler R. Clinical review: Sleep measurement in critical care patients: research and clinical implications. Crit. Care. 2007;11:226.

4. Gujar N, Yoo S-S, Hu P, Walker MP. Sleep deprivation amplifies reactivity of brain reward networks, biasing the appraisal of positive emotional experiences. J. Neurosci. Off. J. Soc. Neurosci. 2011;31:4466-74.

5. Vogel GW, Thurmond A, Gibbons P, Sloan K, Walker M. REM sleep reduction effects on depression syndromes. Arch. Gen. Psychiatry. 1975;32:765-77.

6. Rampil IJ. A primer for EEG signal processing in anesthesia. Anesthesiology. 1998;89:980-1002.

7. Nieuwenhuijs D, Coleman EL, Douglas NJ, Drummond GB, Dahan A. Bispectral index values and spectral edge frequency at different stages of physiologic sleep. Anesth. Analg. 2002;94:125-9, table of contents.

8. Sleigh JW, Andrzejowski J, Steyn-Ross A, Steyn-Ross M. The bispectral index: a measure of depth of sleep? Anesth. Analg. 1999;88:659-61.

9. Iber C, Ancoli-Israel S S, Chesson, AL AL, Quan SF. The AASM Manual for the Scoring of Sleep and Associated Events: Rules, Terminology and Technical Specifications. 1st ed. Westchester, Illinois: American Academy of Sleep Medicine; 2007.

10. Burioka N, Miyata M, Cornélissen G, Halberg F, Takeshima T, Kaplan DT, et al. Approximate entropy in the electroencephalogram during wake and sleep. Clin. EEG Neurosci. 2005;36:21-4.

11. Kirsch MR, Monahan K, Weng J, Redline S, Loparo KA. Entropy-based measures for quantifying sleep-stage transition dynamics: relationship to sleep fragmentation and daytime sleepiness. IEEE Trans. Biomed. Eng. 2012;59:787-96.

12. Brown EN, Lydic R, Schiff ND. General anesthesia, sleep, and coma. N. Engl. J. Med. 2010;363:2638-50.

13. Tung A, Mendelson WB. Anesthesia and sleep. Sleep Med. Rev. 2004;8:213-25.

14. Borbély AA. A two process model of sleep regulation. Hum. Neurobiol. 1982;1:195-204.

15. Borbély AA, Baumann F, Brandeis D, Strauch I, Lehmann D. Sleep deprivation: effect on sleep stages and EEG power density in man. Electroencephalogr. Clin. Neurophysiol. 1981;51:483-95.

16. Carskadon MA, Dement WC. Chapter 2 - Normal Human Sleep: An Overview. In: Dement MHKRC, editor. Princ. Pract. Sleep Med. Fifth Ed. Philadelphia: W.B. Saunders; 2011, $16-26$. 
17. Dahaba AA, Xue JX, Xu GX, Liu QH, Metzler H. Bilateral Bispectral Index (BIS)-Vista as a measure of physiologic sleep in sleep-deprived anesthesiologists. Minerva Anestesiol. 2011;77:388-93.

18. Álvarez-Estévez D, Fernández-Pastoriza JM, Hernández-Pereira E, Moret-Bonillo V. A method for the automatic analysis of the sleep macrostructure in continuum. Expert Syst. Appl. 2013;40:1796-803.

19. Schulz H. Rethinking Sleep Analysis. J. Clin. Sleep Med. JCSM Off. Publ. Am. Acad. Sleep Med. 2008;4:99-103.

20. Tung A, Lynch JP, Roizen MF. Use of the BIS monitor to detect onset of naturally occurring sleep. J. Clin. Monit. Comput. 2002;17:37-42.

21. Noirhomme Q, Boly M, Bonhomme V, Boveroux P, Phillips C, Peigneux P, et al. Bispectral index correlates with regional cerebral blood flow during sleep in distinct cortical and subcortical structures in humans. Arch. Ital. Biol. 2009;147:51-7.

22. Maquet P, Degueldre C, Delfiore G, Aerts J, Péters J-M, Luxen A, et al. Functional Neuroanatomy of Human Slow Wave Sleep. J. Neurosci. 1997;17:2807-12.

23. Nofzinger EA, Buysse DJ, Miewald JM, Meltzer CC, Price JC, Sembrat RC, et al. Human regional cerebral glucose metabolism during non-rapid eye movement sleep in relation to waking. Brain J. Neurol. 2002;125:1105-15.

24. Schuller PJ, Newell S, Strickland PA, Barry JJ. Response of bispectral index to neuromuscular block in awake volunteers. Br. J. Anaesth. 2015;115:95-103. 